\title{
REQUIREMENTS FOR THE ESSENTIAL TRACE ELEMENTS IRON AND ZINC DURING PREGNANCY
}

\author{
Shahla M. Wunderlich \\ Department of Human Ecology \\ Montclair State University \\ Upper Montclair, New Jersey \\ USA
}

The requirements for the essential trace elements iron and zinc are increased during pregnancy in response to fetal and maternal physiological needs. At the same time, the rates of absorption and metabolism of these elements are changing during the gestational period. Low-income pregnant women are often consuming diets that are low in these critical essential elements including iron and zinc. The low intake of these two trace elements is reportedly associated with adverse outcomes including preterm delivery, low birth weight, and maternal morbidity such as vulnerability to infection.

The issues of supplementation of these nutrients during pregnancy are not resolved among scientists. Therefore, the purpose of this paper is to investigate and analyze the current research findings regarding the levels of these nutrients in the diets of pregnant women and to offer guidelines to provide optimal levels these trace elements through foods.

Several studies done by the author indicated that the dietary level of iron and zinc are low. In the most recent study with 249 women, the mean dietary level for iron was $15.6 \mathrm{mg} \pm 7.8$ (Standard Deviation) and zinc was $10.8 \mathrm{mg} \pm 6.3$ (SD). These levels meet $52 \%$ and $72 \%$ of the US Recommended Dietary Allowances for pregnant women for iron and zinc respectively.

Assessment of dietary trace element status must be done in very controlled conditions because the possibility of under or over reporting of serving sizes is a common challenge for nutritionists. Another important factor in this assessment is bioavailabity and cellular metabolism of these nutrients. The dietary intake can only provide the amount of these elements in foods, however, how much of these is actually utilized and metabolized remains uncertain.

Therefore, effective educational measures are necessary to ensure the intake of these nutrients through foods until more is certain about the dietary supplementation of these nutrients. Furthermore, more studies are needed to provide the knowledge of dietary trace element bioavailabity and metabolism during pregnancy from different foods. 